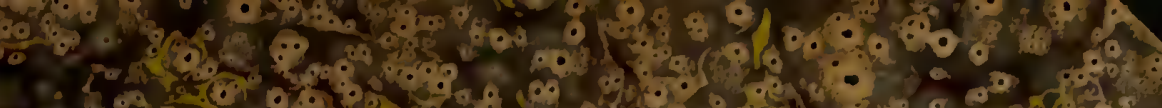

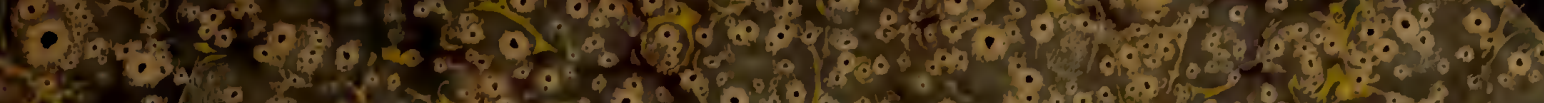

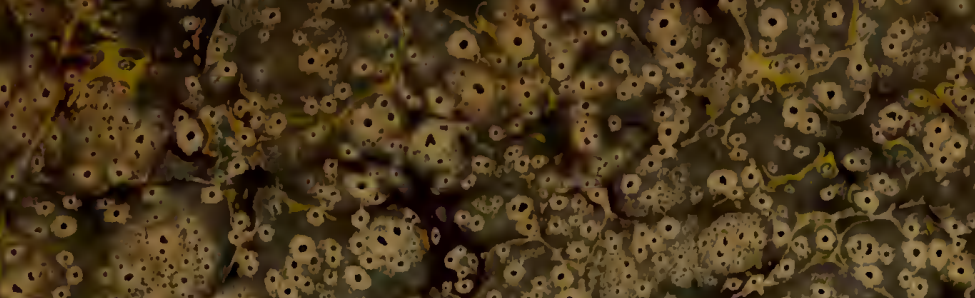

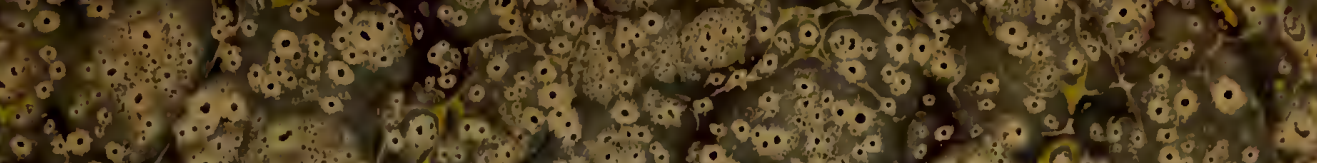

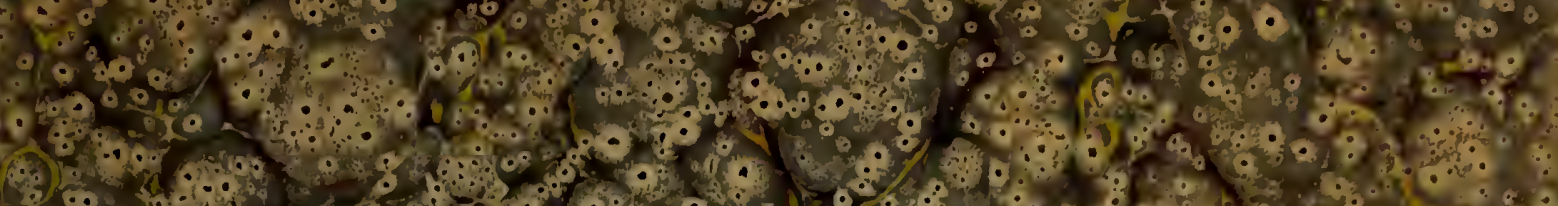

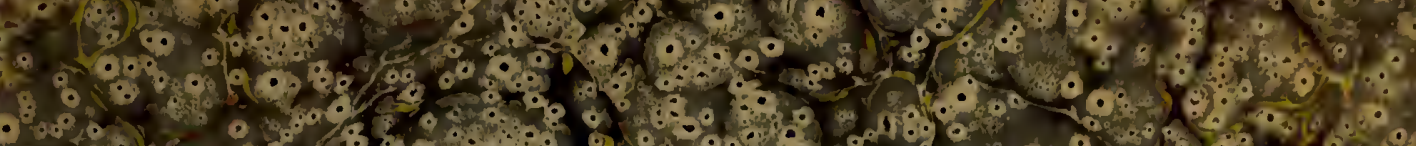

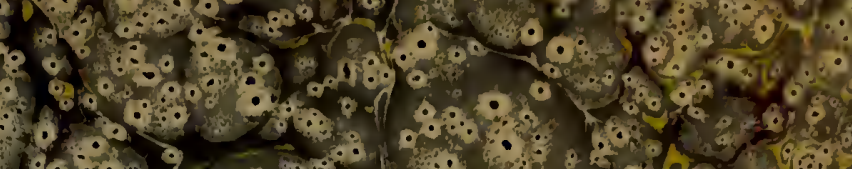

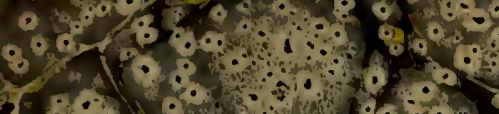

1...

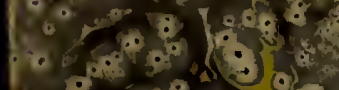

H.

8

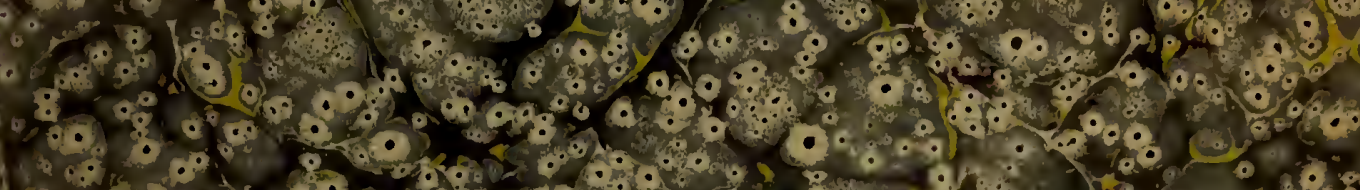

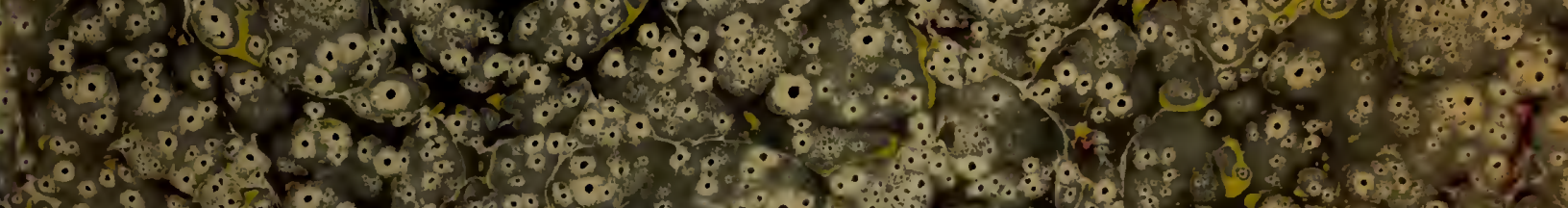

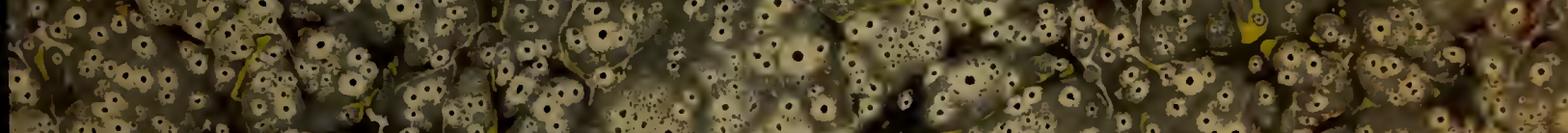

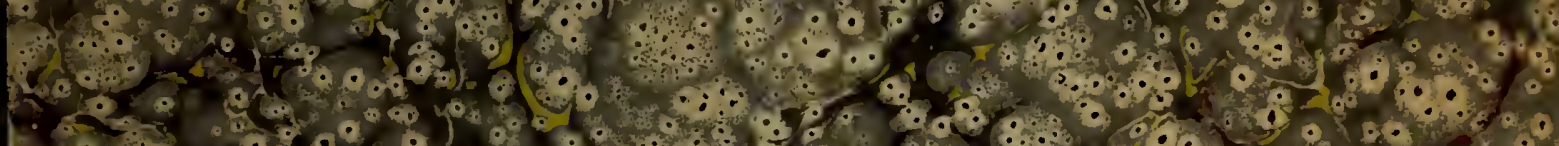

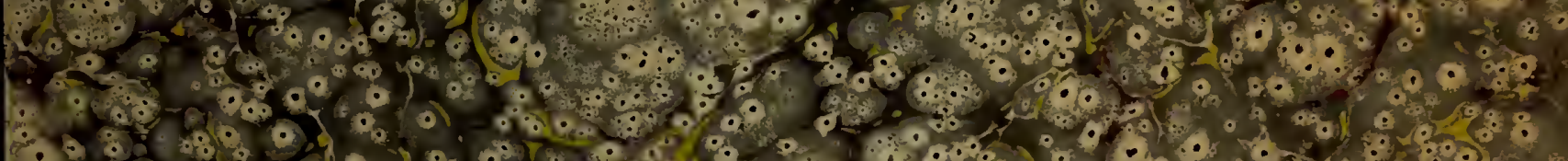

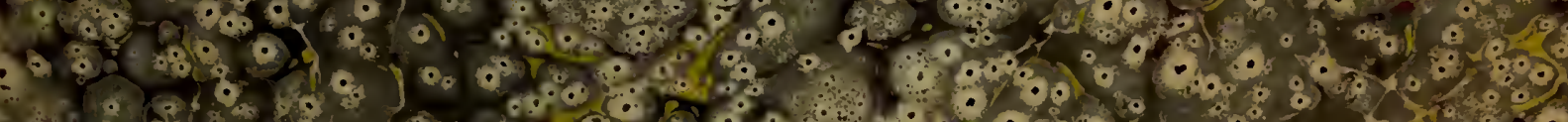

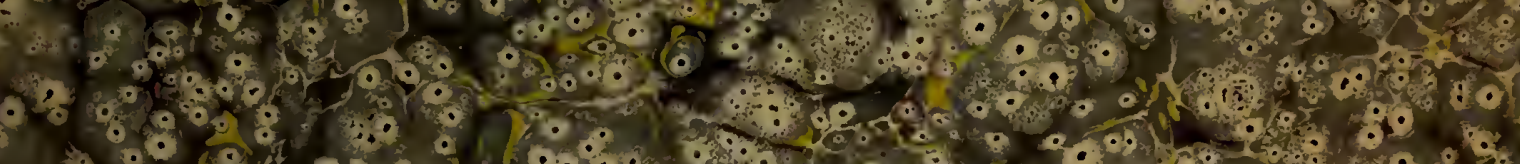

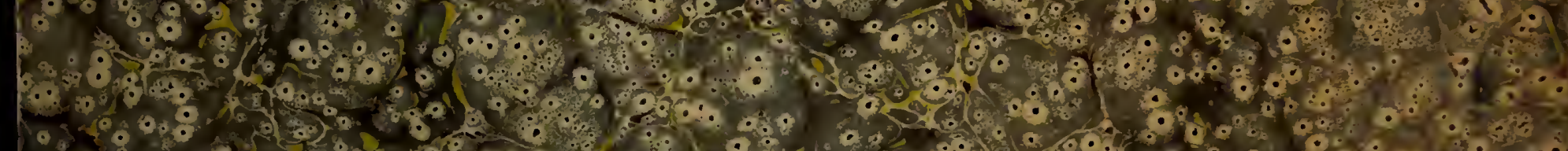

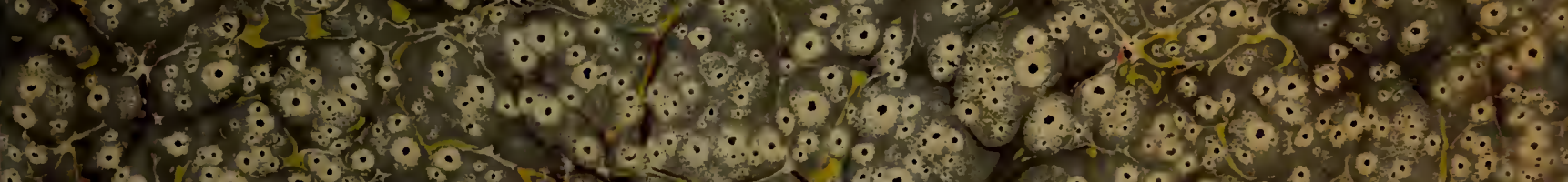

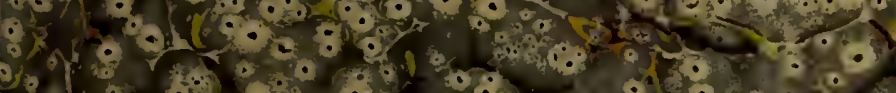

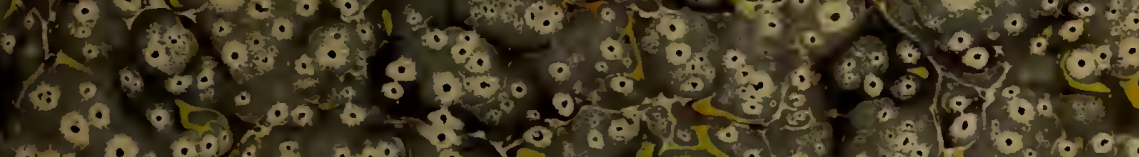

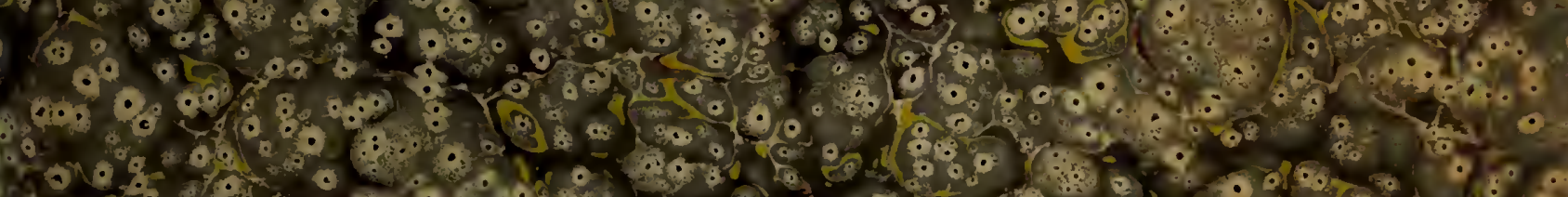

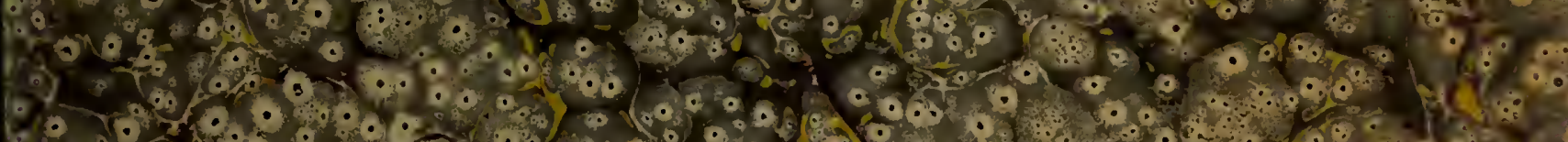

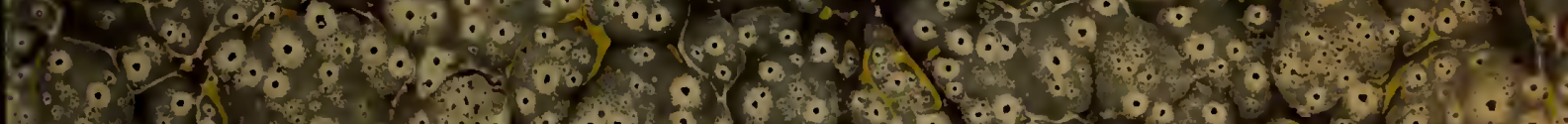

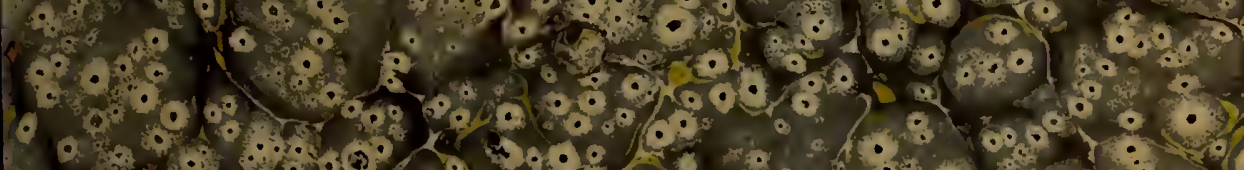

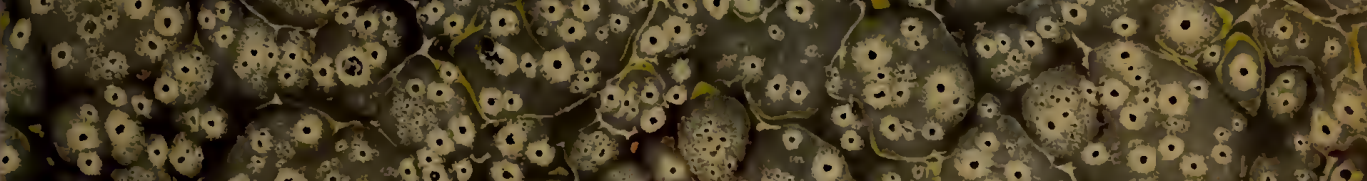

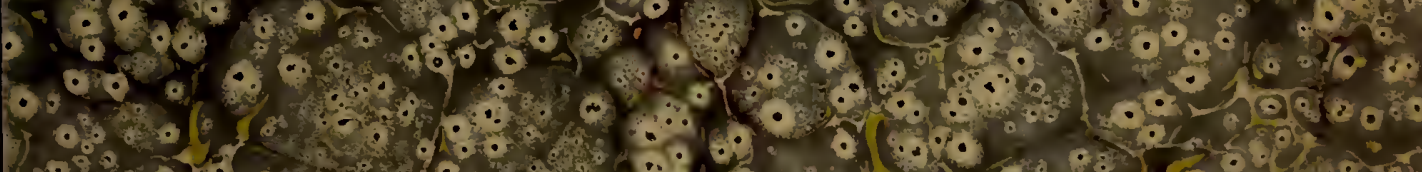

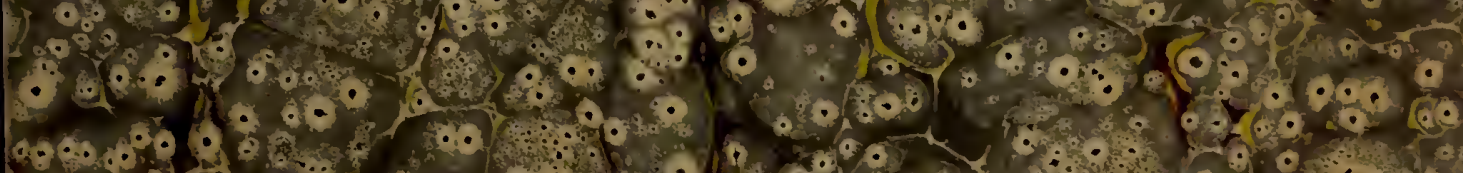

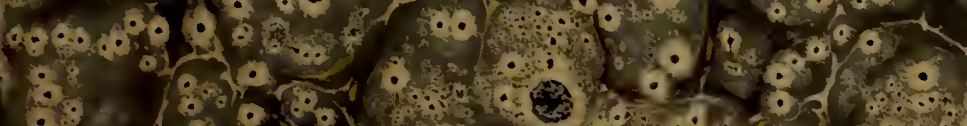

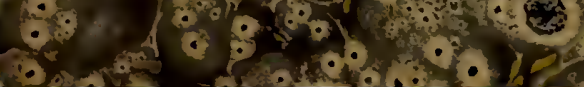



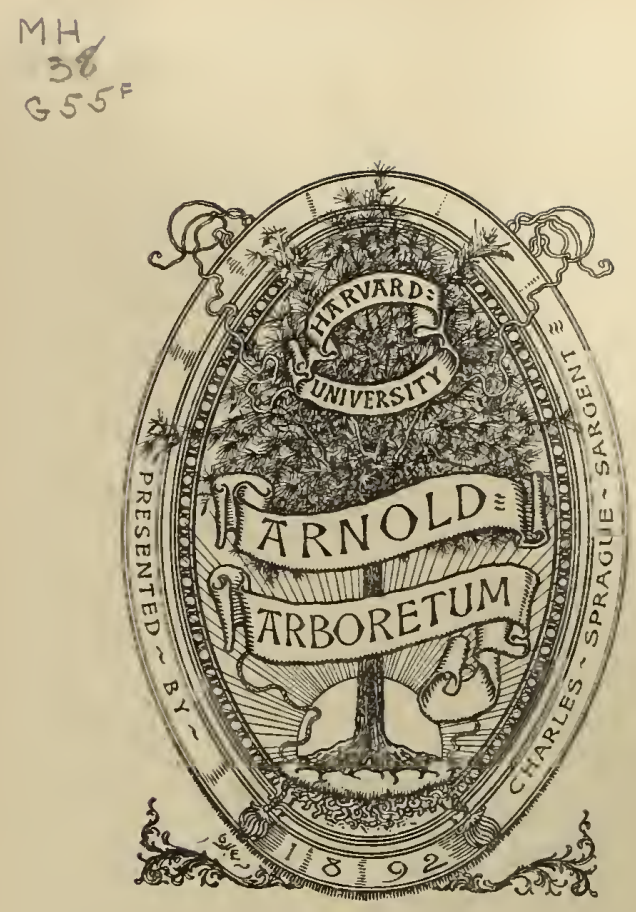








\title{
BEITRÄGE
}

\author{
\%UR
}

\section{KENNTNISS DER DRACÄNEEN}

VON

Dr. HEINRICH ROBERT GÖPPERT,

M. d. A. d. N.

DER FEIER DES FUNFZIGJÄHRIGEN DIENSTJUBILÄUMS DES HERRN CURATORS DER UNIVERSITÄT BRESLAU, GEHEIMEN OBER-REGIERUNGSRATHES HEINKE, GEWIDMET.

\section{BRESLAU, XXXI. OCT. MDCCCLIV.}





\title{
SR. HOCHWOHLGEBOREN
}

DEM KÖNIGLICHEN CURATOR DER UNIVERSIT ÄT,

GEHEIMEN OBER - REGIERUNGSRATHE

\section{HERRN HEINKE}

WÜNSCHT GLÜCK

\section{ZUR FUNFZIG.JÄHRIGEN JUBELFEIER}

\section{SEGENSREICHER AMTLICHER THÄTIGKEIT}

\author{
IN I)ANKBARER ERINNERUNG DER DEN INSTITUTEN HIESIGER KÖNIGLICHER \\ UNIVERSITÄT, ZUMAL DEM BOTANISCHEN GARTEN, GEWIDMETEN \\ FÜRSORGE
}

1)URCII DIESE UNTER VERMITTLUNG DER KAISERL. LEOPOLD.-CAROL. AKADEMIF IER NATURFORSCHER VERÖFFENTLICHTE SCHRIFT

IN TIEFSTER HOCHACHTUNG UND VEREHRUNG

\section{HEINRICH ROBERT GÖPPERT.}

BRESLAU.

DRUCK VON GRASS, BARTH UND COMP. (W. FRIEDRICII). 



\title{
BEITRËCE
}

\author{
ZUR
}

\section{KENNTNISS DER DRACÄNEEN}

VON

\author{
H. R. G O P P E R T, \\ I. d. A. d. N.
}



Zu den Pflanzen, welche gegenwärtig mehr wegen ihres schönen Aeusseren als wegen ihrer Blüthe vorzugsweise kultivirt werden, gehören unter andern auch die Dracäneen. Ungeachtet ihrer grossen Verbreilung in unseren Gärten sind wir jedoch noch weit entfernt, sie alle genau zu kennen. Viele von ihnen lıaben bei uns noch nicht geblüht, daher es nicht möglich war, sie den Gattungen einzureihen, zu denen sie naturgemäss gehören, und andere erscheinen unter so mannigfaltigen Formen, dass auch über ihre Artverschiedenheit noch nicht mit Sicherheit geurtheilt werden konnte. Dies letztere findet sogar bei der interessantesten und uns am längsten bekannten Art der Drachenbäume, bei Dracaena Draco, statt, weswegen ich es nicht für überflüssig hielt, schon im vorigen Jahre auf dies noch zweifelhafte Verhältniss die Aufmerksamkeit der Fachgenossen zu lenken, und jetzt in Folgendem durch Hinzufügung von Abbildungen mich bestreben werde, diese Angelegenlıeit vielleicht zu einigem Abschluss zu bringen. An diese Abhandlung schliesse ich noch ein Namensverzeichniss von 37 gegenwärtig in unsern Gärten kultivirten Arten der Gattungen Dracaena und Cordyline K un th. (enum. plant. V. p. 1-15 et p. 22 -_35), nach Pla ncho n's neuester Bearbeitung derselben, weil solche Verzeichnisse am besten geeignet sind, zu weiteren Forschungen anzuregen. Beigegeben wird noch eine Beschreibung und Abbildung der Cordyline Fontanesiana, welche schöne Art im Juli dieses Jahres im hiesigen botanischen Garten blïhte, und, soviel ich wenigstens weiss, bisher noch nicht 
abgebildet, daher auch vielleicht weniger bekannt geworden ist. Diagnosen sämmtlicher Arten sollen später folgen, wenn es gelungen sein wird, über manche noch zweilelhafte Art oder Form Aufschluss zu erhalten.

\section{Ueber den IDachenbaum, Drucacna Draco I.}

Der Drachenbaum wird nach Alexander v. Humboldt auf Madera und Porto Santo seil den ältesten Zeiten kultivirt, jedoch haben ihn Leopold v. Buch und Berthelot auf Teneriffa auch noch in wildem Zustande angetroffen. Er wächst am üppigsten in den heisseren Gegenden an der Seeküste, doch steigt er wohl noch bis zu 3000 F. Höhe die Berge linan. Nach Berthelot's Beobachtungen ist er im jüngeren Alter etwa bis zu 25 Jahren einfach und von fast gleicher Dicke, erreicht aber of schon eiue Höhe von $50-60 \mathrm{~F}$. In noch höherem Alter trägt er Blüthen und beginnt dann sich zu verästelı. Die bis dahin noch mit fast ringförmigen Narben der über einander liegenden Basen der abgefallenen Bläller bezeichnete Rinde verändert dann auch ihre Beschaffenheit, sie wird mehr lederarlig, endlich rissig und treibt zahlreiche Luftwurzeln. Zwischen den Blallnarben und auch wohl noch an mehreren anderen Theilen des Stammes kommt ein dunkelrothes Harz zum Vorschein, welches eine Sorte des unter dem Namen Drachenblut, Sanguis Draconis, in der Medicin und Technik bekannten Harzes darstelli, die früher auf den Canarischen Inseln häufig gesammelt wurde, jetzt aber nicht mehr von da in den Handel zu gelangen scheint. Unser Baum erreicht ein sehr hohes Alter und einen bedeutenden Umfang. Das kolossalste Exemplar desselben steht in dem Garten des Herrn Franqué in dem Städtchen Orotava auf Teneriffa. Alexander v. Humboldt (Ansichten d. Nat. 2. Bd. S. 104) fand den Umfang desselben mehrere Fuss über der Wurzel 45 P. F. Noch tiefer, dem Boden näher, giebt Le Dru dem Riesenbaume 74 F. Umfang. Nach Georg Staunton hat in $10 \mathrm{~F}$. Höle der Stamm noch 12 F. Durchmesser. Die Höhe ist nicht viel über 65 F. Der Sage nach ward dieser Baum, 
der sicherlich zu den ältesten vegelabilischen Monumenten der Erde gehörı, von den Guanchen, den Ureinwohnern' der Canarischen Inseln, verehrt und in Jahre 1402 bei der ersten Expedition der Bethencourts schon so dick und hohl als jetzl gefunden. Berthelol sagt von diesem Baume: En comparant les jeunes Dragonniers, voisins de l'arbre giganlesque, les calculs qu'on fait sur l'âge de ce dernier effraient l'inagination (Nova Acta Acad. Caes. Leopold.- Carol. nat. cur. T. XIII. 1827. p. 781).

Die erste Kenntniss dieses merkwïrdigen Baumes verdanken wir Clusius, der ihn $\mathbf{1 5 6 4}$ in einem Ḱlostergarten zu Lissabon fand und abbildete (Rar. plant. histor. 1601. Lib. I. Cap. I. p. 1. c. icone). Ob ihn Strabo schon kannte, wie Clusius vermuthet, erscheint ungewiss. *) Clusius führt an, dass er auf den Canarischen Inseln einheimisch und nach Lobel auch in dem Coldenberg'schen Garten zu Antwerpen, so wie auch in dem Grossherzogl. Garten zı Pisa anzutreffen sei. Jonston (Hist. nat. de arbor. Francof. ad Moenum 1662, T. 81) liefert eine Copie der Clısius'schen Abbildung. Linné, der anfänglich ihn auch noclı zweifelhaft zu Asparagus rechnet = Asparagus? Draco, nannte ihn erst in der zwölften Ausgabe seines Systems Dracaena Draco. Die späteren Editoren seines Systems, Willdenow und Persoon, nahmen alle früheren Angaben ïber Dracaena Draco vereint auf, ohne irgend einer Varietät desselben zu erwähnen. Ersı F. G. Hay ne (getreue Darstellung und Beschreibung der in der Arzneikunde gebräuchl. Gewächse, 9. Bd., Berlin 1825, T. 2), welcher die Beschreibungen und Abbildungen des in Rede stehenden Baumes genauer als seine Vorgänger studirte, unterschied drei Abarten: a. strictifolia, $\beta$. laxifolia, $\gamma$. pendulifolia.

*) Clus. I. c. p. 2. Apud veteres neminem islius arboris mentionem facere invenio, nisi forte ea sit, quam Strabo ex Posidonio Gadibus nasci tradit libro Geograplı. tertio his verbis: De arbore Gadibus existente scriptis prodidit Posidonius, quae ramos humum usque incurvatos habeat, frondes saepissine mucronis speciem gestantes, quarum longitudo cubitalis est latitudo digitorum quatuor. Huic et illud innatum esse creditur, ut uno fracto ramo lac effluat, radice abscissa humor exsudet. 
Zu a. strictifolia citirt er die Abbildungen von Clusius (Hist. plant. rar. Lib. I. p. I. cum icone), von Blackwell (t. 358) und Van delli (Dissertat. de Arbore Draconis seu Dracaena 1765 in J. J. Römer scriptor. de plantis Lusit. Hisp. et Brasil. 1796, p. 39-46 (nicht 37, wie Hayne und nach ihm Römer und Kunth angeben). Zu $\beta$. laxifolia eine Abbildung von Crantz (De duabus Dracaen. arbor. p. 25, F. 2. Wien 1768, und Behrens De Dracone arbore Clusii Götting. 1770); jener schreibt er Folia substricta, dieser Folia patenti-pendula zu. $\mathrm{Zu} \gamma$. pendulifolia rechnet er eine andere Abbildung von Crantzl.c. p. 30, F. 3, und Boerha a vi i Ind. alt. plant. hort. Lugdun. T. II. p. 169. Wie sich diese einzelnen von Ha y n e citirten Abbiloungen zu einander verhalten, habe ich schon früher ausführlich in der Flora (1853, p. 394u. ff.) auseinandergesetzt. Zwei Jahre vor Hayne, was ihm unbekannt geblieben war, sah sich Tenore, gestützl auf dieselben Autoren (Tenore ad Florae Neapolit. Prodr. app. IV. 1823), veranlasst, unsere letzterwähnte Varietät als eigene Art unter dem sehr passend gewählten Namen Dracaena Boerhaavii aufzustellen, welche jedoch Römer und Schultes (Röm. et Schult. system. VI. p. 339) nicht anerkennen und ebensowenig $\mathbf{K}$ unth (enum. V. p. 3, 1850), obschon Tenore 1845 (Catalogo del real orto botanico di Neapoli p. 84, 85) abermals auf den Unterschied derselben von Dracaena Draco aufmerksam machte und sie folgendermaassen charakterisirte:

\section{Dracaena Boerhanvii 'Tenore.}

„DD. caudice arboreo, foliis loratim lanceolato-linearibus praelongis flaccidis, spiraliter contorlis apice inermibus; floribus subcampanulatis, pedunculis geniculatis longioribus filamentis medio crassioribus; panicula terminali erecta. Tenore, Atti della Reale Accademia delle scienze di Napoli tom. 3, p. 37, tav. 3; Cordyline foliis inermibus integerrimis, flaccidis Royen Hı. Lugd. Bat. p. 22; Palma loliis longissimis pendulis e caudice glabro enatis. Boerh. Lugd. Bat. 2, p. 160.66 
,Obs. D. Draconis nomine a planlarum mercatore Camberiensi Martin Burdin, anno 1814, salis parvulam accepi. Florentem vero in II. R. N. elapso anno (1821) habui, cum vix ad septem pedum altitudinem se exlulerit. Cum vero D. Draconis descriptiones synonymiasque apud auctores diligenter perscrutaverim, sub eodem D. Draconis nomine in horlis duas distinclas latuisse species suspicatus sum. Absurde nempe foret eandem plantam Boerhaavium Royenumque foliis flaccidis longissimis inermibus, Linnaeum cacterosque vero foliis erectis spinosis dixisse. ${ }^{6}$

„Avendomi successivamente procurata la vera D. Draco, entrambe le piante vegetano di presente nella slufa temperata del Real Orto. Chiunque vorrà darsi la pena di guardarle, non potrà astenersi dal riconoscerne la diversità di specie, non essendo ragionevol cosa il ritenere la D. Boerhaavii qual varietà della D. Draco come han fatto diversi autori. (Tenore am angeg. 0. $)^{\text {c: }}$

Ich kann mich nur der Ansicht von Te nore anschliessen, denn wel die Dracaena Draco $\gamma$. pendulifolia, welche eben der Dracaena Boerhaavii entspricht, mit der Dracaena Draco a. strictifolia oder der wahren Dracaena Draco Tenore genauer vergleicht und namentlich in ihrer Entwicklung zu beobachten Gelegenheit hat, wird sich schwerlich für die Identität dieser beiden Pllanzen erklären. Junge, dreijährige, aus Samen des botanischen Gartens zu Zürich gezogene Pflanzen, die ich für die echte Substricta halte, entsprechen ganz und gar der von Berthelot (Observ. sur la Dracaena Draco in N. Act. Ac. C. L.-C.XV. p. 773, T. 35, F. 1 u. 2) im Uıriss gegebenen Abbildung und der oben erwähnten Beschreibung des trefflichen Clusius. Mein hochgeschätzter Herr College Oswald Heer bestätigel diese Meinung, indem er diesen Samen aus dem Vaterlande des Drachenbaums von den Canarischen Inseln mitgebracht hatte, wo er nur die Form mit steifen Blätlern beobachtete, während die mit schlaffherabhängrenden Blältern ilın nur in Europäischen Gärlen vorgekommen war. Unsere jungen Pllanzen ähneln in der Thal, wie Clusius sagt, einer Iris und ihre mit einander gleichen und parallelen Nerven durchzo- 
genen Blätler sind etwa 1' lang und 2" breit, steif, nicht etwa wellig, an der dem Stengel zugekehtlen Seite wie auch am Rande roth und elwas graugrün, an der Spitze jedoch schon elwas rinnenförmig und zusammengerollt. Inzwischen sehe ich, dass der rothe Rand bei den vorjährigen Blättern sich schon anfängt zu verlieren; eine durchsichtige weisse Einfassung bleibt zurück, die sie dann beibehalten. Berthelot's Uimrisszeichnung lässt zwar in den Blätlern der einen Figur einen Mitlelnerven erkennen, da er ihn aber in der Beschreibung nicht erwähnt, so ist dies wohl nur einem Versehen des Zeichners zuzuschreiben. Als Repräsentant der erwachsenen Pflanze, ganz eben so wie sie auf Berthelot's Taf. XXXV abgebildet ist, kultiviren wir ein prächtiges, bis zu der Blattkrone 6' und inclusive der Blattkrone 9' hohes Exemplar, welches, als es im Jahre 1818 unter der Direction meines verehrten Lehrers und Freundes L. C. Treviranus, des Begründers der Flora des hiesigen botanischen Gartens, aus dem Garten des Belvedere bei Weimar erworben wurde, schon Manneshöhe hatte, also bei so langsamem Wachsthume gewiss schon ein Altel von nahe an 100 Jahren besitzen mag. Die unteren $3^{\prime}$ langen Blälter der schönen 6 - 7 ' breiten Krone hängen bogenförmig herab, die in der Mitte stehen wagrecht, die innern oder obern 1-3jährigen aufrecht; alle aber sind steif, flach, nicht wellig, weniger graugrün als die der jungen Pflanze, und am Rande nicht roth, sondern mit jener obenerwähnten zarlen durchsichtigen Einfassung versehen, aber an der Spitze elwas rinnenförmig zusammengerollt, überhaupt ganz so, wie sie an den angezeigten $0 r-$ ten beschrieben wurden. *) Die Ansätze der Blätler sind 4" breit, die Blätter selbst an der Basis von 3/" Breite, überhaupt von mehr dunkelgrüner Farbe als die erwähnten jungen Pllanzen. Auch hier zeigen sie schon einen überaus strafferen Habitus, wie auch aus der von der Meisterhand

*) Hr. Prof. Dr. Oswald Heer (Der Rentongarten zu Funchal, Garlenflora von E. Regel. 1. Jahrg. Erlangen 1852 , S. 23) sagt auch, dass die Blätter an den Aesten des Drachenbaumes etwas schief stehen. 
unseres Weitz gelieferten Abbildung hervorgeht, die ich auf Taf. I. Fig. 1 beifügre. Sie stellt den oberen Theil des eben erwähnten Exemplars unseres Gartens in verjüngtem Maassstabe, etwa im 18ten Theile der natïrlichen Grösse dar.

Ganz anders verhalten sich die Pllanzen, welche eben der Form pendulifolia oder der Dracaena Boerhaavii $\mathrm{T}$ e $\mathrm{n}$. entsprechen, die wir vor drei Jahren aus Samen von Padua erzogen. Schon im ersten Jahre legten sich die durchweg grïnen, nur an der dem Stengel zugekehrten, ihn umfassenden Basis röthlichen Blätter zurück, noch ehe sie sich völlig entwickelt hatlen, so dass sie nicht bogenförmig herab, sondern mit dem Stamme parallel hingen. Alle waren und sind noch jelzt schlaff, $2-\mathbf{3}^{\prime}$ lang, am Rande wellig, aber an der Spitze doch steif und wie die Blätter der ersteren Form etwas rinnenartig zusanmengerollt und daher stechend. Ebenso erscheinen die Blätter mannshoher mit schönen Kronen versehener Stämme. Fast alle hängen schlaff herab und sind ganz so, wie Te nore es angiebt, fast spiralig gerollt, aber an der Spitze wie die jüngeren ebenfalls etwas zusammengerollt und somil stachelspitzig, daher ich in dieser Hinsicht mit dem von Tenore angegebenen Charakter, ,apice inermia ${ }^{66}$ nicht übereinstimmen kann. Alle diese Kennzeichen insgesammt verleihen Stämmen dieser Art ein völlig verschiedenes Aussehen. Die Breite ihrer Kronen ist viel geringer als der Längendurchmesser, während bei der Form striclifolia oder der wahren Dracaena Draco die Kronen fast gleich breit und hoch sind. Auf Taf. II. Fig. 1 liefere ich eine Abbildung der eben genannten dreijährigen Pflanze in $\%$ der natürlichen Grösse. Im Allgemeinen ist die Dracaena Boerhaavii oder die Form pendulifolia in Gärten häufiger als die wahre Draco, wie ich denn in Berlin und Hamburg nur die erstere glaube gesehen zu haben. Die botanischen Gärten in Göttingen, Halle, Leipzig und Dresden, und nach den Mittheilungen meines Collegen, Herrn Geh. Medicinalraths Prof. Dr. Betschler, eines genauen Kenners der Dracäneen, auch der zu Wien, wie die Gärten Belvedere bei Weimar, enthalten schöne Exemplare der Dracaena Draco; das stärkste befindet sich 
zu Göttingen. Römer und Schultes fügen den obigen drei auf Hay ne's Autorität aufgenommenen Varietäten noch eine vierte hinzu: $\delta$. angu stifolia, und citiren hierzu Jacq. Fragm. 2, p. 4. T. II., F. 4, welche auch $\mathrm{Kunth}$ annimmt, die aber keine andere, als eine von $\mathrm{Crantz}$ angefiihrte mehr schmalblättrige Form, seine Oedera dragonalis ist, welche aus den anderweitig angegebenen Grïnden (Flora 1853, S. 398) zu Dracaena Boerhaavii gehört.

Zunächst wäre noch zu ermitteln, wo eigentlich die Dr. Boerhaavii herstanmt. Crantz giebt für Stoerkia Draco (Dracaena Draco) die Canarische Insel Porto Bello, für die zweite oder unsere Art ebenfalls eine Canarische Insel, aber Madera als Vaterland an. Herr Prof. Dr. He er, der jüngst erst diese an Pflanzen so reichen Gegenden besuchte, hat daselbst, wie ich schon erwähnte, nur Dr. Draco gesehen, und kennt Dr. Boerhaavii nur in Europäischen Gärten, wo sie sich übrigens auch schon aus Samen fortgepflanzt hat, wie mir Herr Inspektor und Gartenmeister Wendland zu Herrenhausen güligst mittheilte, der aus einem im Jahre 1820 fruktificirenden Exemplare Pflanzen erzog, welche eben ganz und gar der Mutterpflanze der Dr. Boerhaavii entsprechen.

Uebrigens wurden beide Arten nicht blos dort in jenem wabren Emporium von Prachtpflanzen aller Art, sondern auch in vielen andern Gärten ohne Beeinträchtigung ihrer Eigenthümlichkeiten in einer und derselben Temperatur seit Jahren kultivirt, was ich schliesslich nur noch anführe, wenn man etwa geneigt sein wollte, die schlaffere Haltung der Blätter auf Rechnung wärmerer Kultur zu setzen, wie dies hie und da bei andern Pflanzen wohl beobachtet worden ist. Dracaena Boerhaavii wächst rascher als die Dracaena Draco.

Schliesslich folgt nun mit Rücksicht auf die angeführten Beobachtungen die Diagnose der beiden Arten nebst ihren Synonymen. 


\section{Wracuena Draco E. ex parte.}

Dr. arborea; apice ramosa, foliis sessilibus semiamplexicaulibus linearibus apicem versus sensim altenuatis apice ipso canaliculatis spinescentibus planis inferioribus arcuatim dependentibus, mediis patentibus, summis erectis; paniculis terminalibus ramosis foliaceo-bracteatis (ramis ternis patentissimis, floribus quaternis el quinis).

Draco arbor Clusius hist. pl. rar. 1. 1 p. 1 c. icone.

Bauh. Pinax p. 505, Blackw. herb. T. 358.

Arbor Draconis, Draco yuccaeformis vel Dracaena. Vandelli Dissert. in Römer script. de plant. Hisp., Lusit., Bras. p. 37, T. $2 a b$.

Stoerkia Draco Crantz de duab. Dracon. arbor. p. 25, F. 1, 2.

Arbor Draconis latifolia Hortulan. fide Crantzii p. 21.

Yucca Draconis Hoyer Amoenit. acad. Linn. III. p. 407.

Asparagus? Draco Linn. sp. pl. edit. 2, T. 1, p. 451.

Dracaena Draco Linn. Syst. Nat. edit. 12. II. p. 246; Willd. sp. pl. II. p. 155; Haw. pl. succ. p. 30; Ej. Syn. p. 67 ; Lam. Enc. meth. II. p. 323 ; Dalm. diss. praes. Thunb. p. 3; Gleditsch in Act. Acad. Scient. Berol.; Behrens dissert. Gölting. 1770, p. 36, F. 1, 2, 3; Meyer in Mém. de l'Acad. royale de Berlin 1796 et Berlin 1799, p. $29-44$ avec pl.; Berthelot in Nova Acta Acad. Caes. Leop. Car. n. cur. XV. p. 773, T. 35-39; Dra-

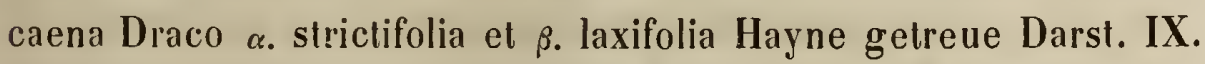
T. 2; Röm. et Schult., syst. veg. T. VII. 1. p. 37; Kunth enum. T. V. p. 3.

\section{Dracaena Boerhanvii Tenore.}

Dr. arborea; apice ramosa, fol. sessilibus semiamplexicaulibus linearibus sensim attenuatis apice canaliculatis spinescentibus junioribus et adultis laxis flaccidis undulatis dependentibus; paniculis terminalibus erectis (floribus subcampanulatis pedınculis geniculatis longioribus, filamentis medio crassioribus). Tenore Atti della reale Acad. delle science di Napoli 
T. 3, p. 37, tav. 3 ; Ej. fl. neap. prodr. App. IV., Catal. del real orto botanico di Napoli 1845 , p. 85.

Cordyline fol. inermibus integerrimis flaccidis Royeni Lgd. bat. p. 22. Palma foliis longissimis pendulis e caudice glabro enatis Boerl. Lugd. Bat. 2, p. 160.

Oedera dragonalis Crantz 1. c. p. 30, F. 3.

Arbor Draconis angustifolia Hortul. fide Crantzii p. 28.

Dracaena Draco pendulifolia Hayne l. c.; Römer et Schult. syst. veg.

T. VII. 1. p. 338; Kunth enum. T. V. p. 3.

Dracaena Draco $\delta$. angustifolia Jacq. Fragmenta 2, p. 4, t. 2, F. 4.

\section{Verzeichniss}

der

gegenwärtig in Europ. Gärten kultivirten Arten der Gattungen Dracaena und Cordyline Kunth, welche sich sämmtlich in der Privat - Sammlung des Geheim. Medicinalrathes Hern Professor Dr. Betschler und im hiesigen botanischen Garten befinden.

\section{Dracaena $\mathbf{H}$.}

1) Dracaena Draco L. ex parte. Syn.: Dracaena canariensis Hort. Canarische Inseln.

2) - Boerhaavii Tenore.

Dem Habitus nach glaube ich zu Dracaena rechnen zu können :

3) - Ehrenbergii G. Fintelm. Pfaueninsel bei Potsdam. Mexiko?

4) - longifolia Hort. Belg. (Makoy).

- frutescens französischer Gärten, ist mir unbekannt, ebenso Dracaena gracilis Hort. Hoult., Dr. amaryllidifolia et Dr. undulata Hort. Baum. Bollwill. 
Dracaena Dioscoridis Hort. Belg., bekannter unter dem mir seiner Abstammung nach gänzlich unbekannten Namen Pincinecticia oder auch Pincinectia. P. tuberculata gehört schwerlich zu den Dracäncen und eben so wenig die drei anderen Arten dieses Geschlechtes, P. glauca, gracilis und linifolia der belgischen Gärten. - Mexiko?

\section{Cordylime Commers. Planch. FI. des Serres et des Jard. d'surope 1850,51, p. 136, 38.}

1) Cordyline reflexa Planch. I. c. Syn.: Dracaena reflexa Lam. Redouté Liliac. T. 92 et Kth., Dr. purpurea Ht. Berol., Dr. cernua Ht. Berol. nec. Jacq. - Ins. Madagascar, St. Helena.

2) - salicifolia nob. Syn.: Dracaena linifolia Hort.; Dr. salicifolia Ht. Berol.? - Java?

3) - madagascariensis nob. Syn.: Dracaena madagascariensis $\mathrm{Ht}$. Belg. (Makoy).

Eine höchst ausgezeichnete Art.

4) - cernua Planch. I. c. Syn.: Dracaena cernua Jacq. Hort. Schönbr. 1, 50, F. 96. - Insel St. Mauritii.

5) - Rumphii Hook Syn.: Dracaena angustifolia Roxb. fl. ind. 2, p. 155. - Amboina und Java.

In Gärten selten in blühenden Exemplaren, unter andern ausser im Berliner botanischen Garten, bei H. Hage in Erfurt und in Herrenhausen.

6) - fruticosa nob. Syn.: Dr. fruticosa Ht. Berol. Von Kunth zu der vorigen Art gezogen, weicht sie jedoch, wie blühende Exemplare zeigten, wesentlich von ihr ab. Ich werde sie später ebenfalls abbilden."

7) - fragrans Pì. 1. c. Syn.: Dr. fragrans Gawl. im Bot. Mag: T. 1081. Aletris fragrans L. - Guinea, Sierra Leone. 
8) Cordyline Sieboldii Pl. 1. c. p. 109 et p. 136 Syn.: Dracaena javanica Kunth.

3. maculata Pl. 1. c. p. 109, T. 569. - Java.

9) - Fontanesiana PI. l. c. Syn.: Dr. elliptica Desf. Cat. 388; Dr. nigra Ht. Berol.; Dr. Fontanesiana Schult. System. 7. 1676. - Insel Bourbon.

Abgebildet auf Taf. III. Fig. 3, nach einem im Juli d. J. blühenden Exemplare. Der Stamm nach Desfontaines baumartig, bei uns nur 26 " hoch, $4-5$ Linien dick, mit 10" langer Blüthenrispe. Die Blätter (a) länglich, 7-8" lang, 2-2 $\frac{1}{2}$ " breil, etwas zugespitzt, dick, fast lederartig, von dunkelgrüner Farbe, oberhalb glänzend, unten matt, mit 8 bis 9 besonders hervortretenden parallelen Längsnerven, übrigens ganzrandig. Die Blüthenrispe endständig, 10" lang, aufrecht, mil einigermaassen hin- und hergebogener und mit Längsfurchen versehener Spindel. Die abwechselnd gestellten Aeste unterhalb fast horizontal abstehend, an der Basis mit $1-1 \frac{1}{2}$ " langem und 1 " breitem Deckblatte (b) versehen. Die Blüthen (Fig. 4) büschelförmig, zu drei, sehr kurz gestielt mit einem gemeinschaftlichen Deckblätlchen. Die einzelnen Blüthen (Fig. 5) röhrig, 8-10"' lang, etwas gebogen, bis zur Mitte 6theilig. Fig. 6 stellt eine schwach vergrösserte, Fig. 7 eine geöffnete Blïthe dar; die Abschnitte linear (a), nach innen hohl mit einem nach innen geschlagenen Ende, übrigens von der Länge der Blüthenröhre. Die Staubgefässe (b) mit grünem Träger und gelblicher tief 2 theiliger Anthere. Fig. 8 die Anthere von vorn und Fig. 9 die Insertion des Trägers. Das Pistill Fig. $7 c$ mit länglichem, in den Griffel $d$ sich allmälig verdünnendem Ovarium; $e$ die dreilappige Narbe, welche Fig. 10 noch etwas vergrössert dargestellt erscheint; die einzelnen Lappen 
der Narbe rundlich. Die Samen kamen nicht zur Entwicklung. Sie waren zu $\mathbf{1}-\mathbf{2}$ in jedem Fache des dreilächrigen Fruchiknotens sichtbar.

10) Cordyline ovata PI. 1. c. Syn. : Dracaena ovata Gawl. Bot. Mag. T. 1180 , Kunth 1. c. - Sierra Leone.

11) - umbraculifer a nob. Syn.: Dracaena umbraculifera Jacq. Schönbr. 1, 50, T. 95; Kunth I. c. p. 8. - Vaterland unbekannt, kultivirt in Ostindien, Java, St. Moritz.

Gehört nach Untersuchung getrockneter, im Königl. Central-Herbarium zu Schöneberg befindlicher, so wie frischer blühender Exemplare zu Cordyline.

Nach dem Habitus rechne ich bis zur Beobachtung der mir bis jetzt noch nicht bekannten Blüthen vorläufig hierher noch folgende Arten:

12) - nutans Hort. Ob es die von Cunningham beschriebene Art dieses Namens isi, kann ich nicht behaupten.

13) - coerulea Hort. Aus dem Garten zu Tetschen von Hrn. Josst.

14) - Hooibrenkiana nob. Wie die beiden folgenden aus den reichen Sammlungen des Hrn. Hooibrenk zu Hitzing bei Wien. Syn.: Dracaena selandica Hooibrenk.

15) - humilis nob. Syn.: Dracaena humilis Hooibrenk.

16) - Betschleriana nob., als Dracaena arborea vera von Hrn. Hooibrenk. Durch die an der Basis 1 $1 \frac{1}{2}-2^{\prime \prime}$ breiten, lang zugespitzten, 1-1 1\%' langen, fast aufrecht stehenden ganzrandigen, dunkelgrünen, aber roth eingefassten Blätter sehr ausgezeichnet.

17) - arborea nob. Syn.: Dracaena arborea Link. enum. Kunth 1. c.; Aletris arborea Willd. - Afrika.

18) - marginata nob. Syn.: Dracaena marginata Lam. Encyclop. 2, p. 324, Kunth 1. c. p. 8; Dr. tessellata Willd. enum. 
374; Dr. mauritiana Hort. Berol. 1841, nec Dr. mauritiana Willd. Herb., quae ad Cohniam floribundam Kunth pertin. (Dr.marginata Ait. Kew. 1, 454 est Lomatophyllum borbonicum Willd.).

B. concinna nob. Syn.: Dracaena marginata latifolia $x$. concinna Kunth et Ht. Berol. - Ins. Madagascar, Bourbon.

19) Cordyline cannaefolia Brown. Prodr. 280, Schult. Syst. 7, 347, Kunth et Planch. l. c. - Tropisches Neuholland. Von dieser immer noch nicht hinreichend bekannten Art, deren Habilus mehr den Arten von Calodracon als Cordyline gleicht, befindet sich ein baumförmiges Exemplar in Herrenhausen, dessen Blüthenentwicklung hier also Entscheidung liefern kann.

\section{Iracaemopsis Planch. I. c. p. 110 et $13 \%$.}

1) Dracaenopsis australis Pl. I. c. Syn.: Dracaena australis Hook.; Dr. obtecta Grah. fide Hook.; Cordyline australis Kunth I. c. p. 29. - Insel Norfolk, Neuseeland und Neuholland.

2) - indivis a PI. I. c. Syn.: Dracaena indivisa Forst pl. escul. n. 33 ; Cordyline indivisa Kunth enun. l. c. p. 30. - Neuseeland.

Diese neuseeländische Pflanze soll sich unter dem Namen Cordyline australis, Dianella australis und Freycinetia Baueriana in unseren Gärten befinden, was mir noch weiterer Aufklärung zu bedürfen scheint.

\section{Calodracon Planch. I. c.}

1) Calodracon Jacquinii Pl. l. c. Syn.: Cordyline Jacquinii Kunth. - China. 
In Gärten zwei Varietäten :

a. atrosanguinea nob., mil gleichförmig dunkelblutrothen Blättern. Dracaena ferrea L. Syst. n. 275, Bot. Mag. T. 2052 el hortor.

ß. purpureo-variegata nol., mit grün- und purpurfarbenen Blättern. Dracaena terminalis Hort. nec Reichard.

2) Calodracon heliconiaefolius Pl. I. c. Syn.: Cordyline heliconiaefolia 0tto et Dietr. in Berl. Allgem. Gartenzeit. 1835, n. 4, p. 32, n. 25, p. 231 ; Kunth Acl. Acad. Berol. 1842, p. 30 el ej. enum. V. p. 28; Dracaena brasiliensis Hort. Aus China in Brasilien eingeführt.

3) - Sieboldii PI. I. c. Syn.: Dracaena nobilis Ht.; Dracaena Sieboldii Ht. v. Hoult. - Japan.

\section{Charlmoodia Sweet. et Planch. I. c. p. 138.}

1) Charlwoodia congesta Sweet. fl. Austr. T. 18, Syn.: Dracaena congesta Sweet. Hort. Brit. 424; Cordyline congesta Kunth I. c. - Neuholland.

2) - stricta Sweet. fl. Austr. Fol. 18, p. aversa Syn.: Dracaena stricta Bot. Mag. T. 2575; Cordyline stricta Kunth I. c. - Neuholland oder Neuseeland.

3) - spectabilis Pl. I. c. Syn.: Cordiyline spectabilis Kunth et Bouché in Ind. sem. Ht. Berol. 1848; Cordyline dracaenoides Kunth et Acl. Acad. Berol. 1842, p. 30; Dr. stricta Ht. Berol. nec Sims. - Vaterland?

4) - angustifolia nob. Syn.: Cordyline angustifolia Kunth enum. 5, p. 32 ; Dracaena paniculata Ht. Berol. 1847. Vaterland?

5) - rubra PI. l. c. Syn.: Cordyline rubra Hügel in Ht. Berol. Kunth enum. p. 35. - Vaterland? 
$18(58)$

II. R. Göppert,

6) Charlwoodia fragrantissima Lemaire le Florist. Vol. IV. PI. 399, Oct. et Nov. 1853. - St. Paul in Brasilien.

7) - ens ata nob. Syn.: Als Dracaena ensata, von Hrn. Hooibrenk, doch wohl schwerlich Dracaena ensata Thunb. in Dalman. Diss. 3; Kunth 1. c. p. 15.

8) - longifolia nob. (Ht. Belgic. Makoy).

9) - australis nob. Als Species nova aus Neuseeland, im Garten des Hrm. Hooibrenk. 
Beilräge aur Kemulniss der Dracäncen.

(59) 19

\section{Erklärung der Tafeln.}

\section{Tafel I.}

Fig. 1. Dracaena Draco L. ex parte.

Tafel II.

Fig. '2. Dracaena Bocrhaavii Tenore.

Tafel III.

Fig. :3 bis 10. Cordyline Fontanesiana Planch. 


4

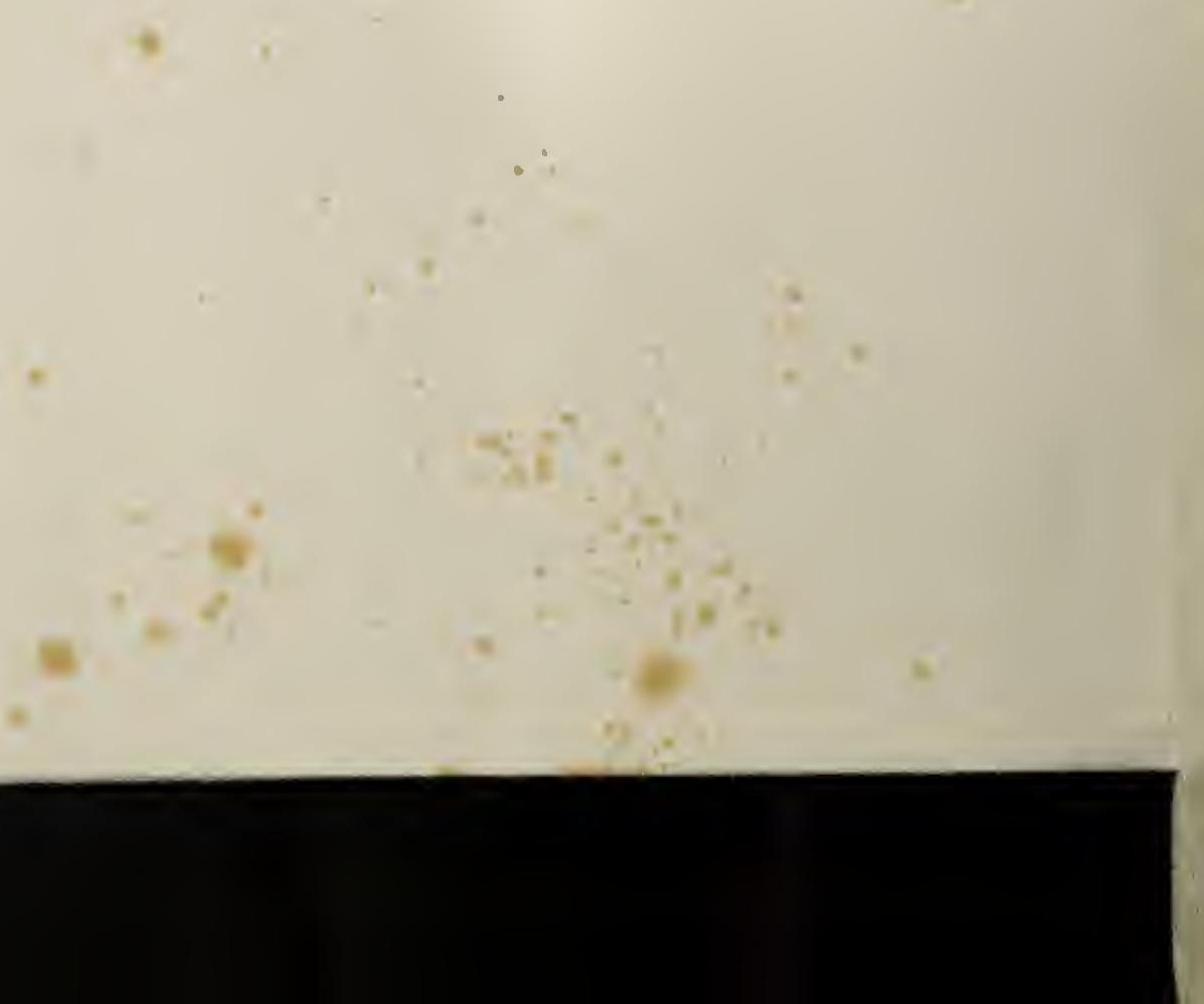






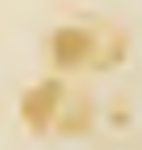

$+n^{2}$

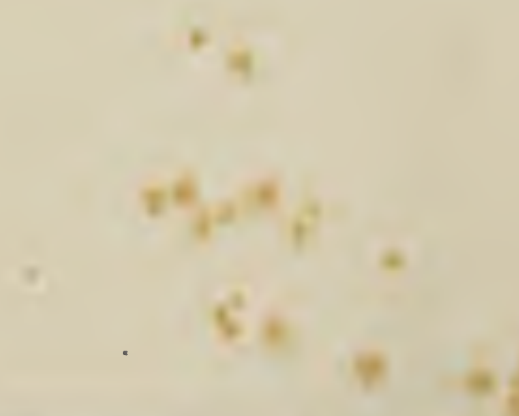







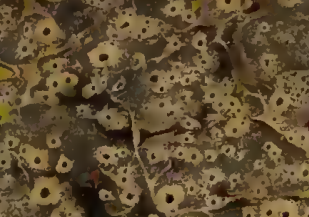

20
3

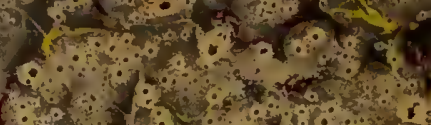

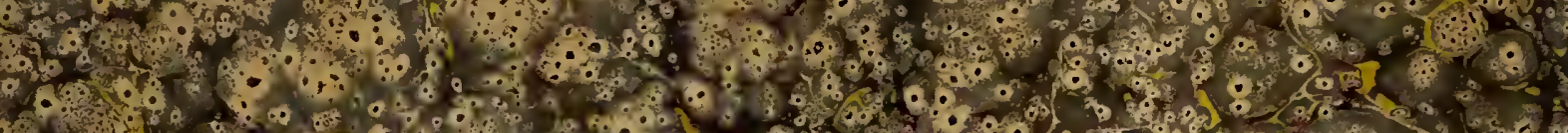

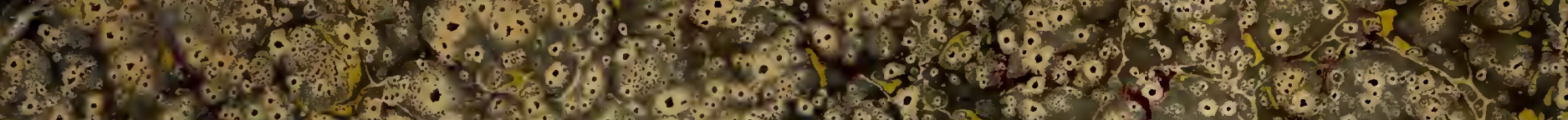

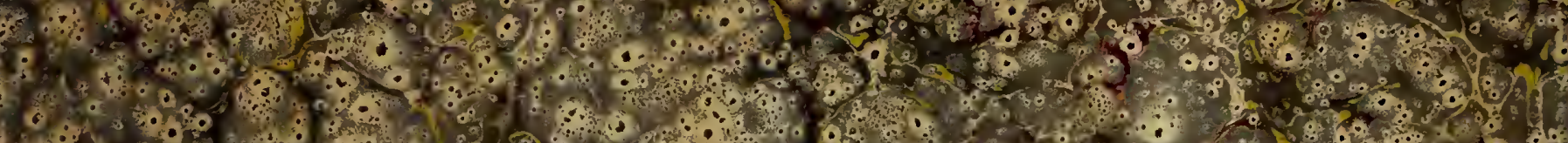

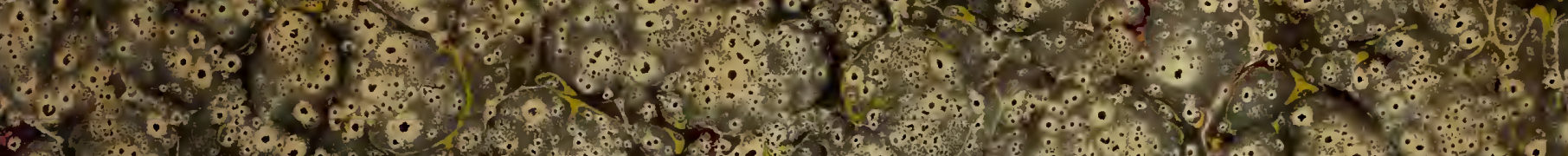

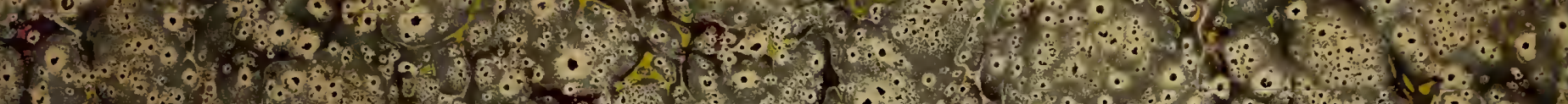

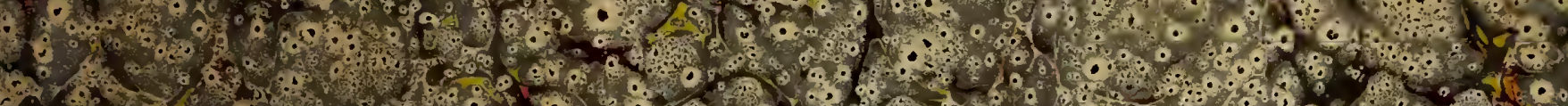

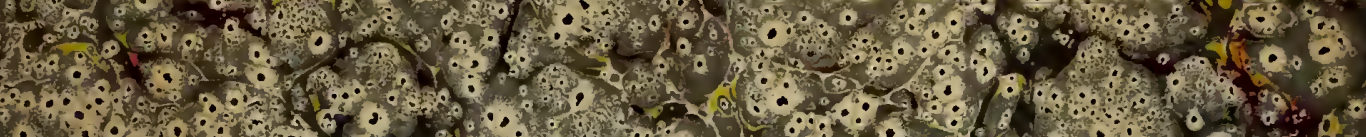
(5.

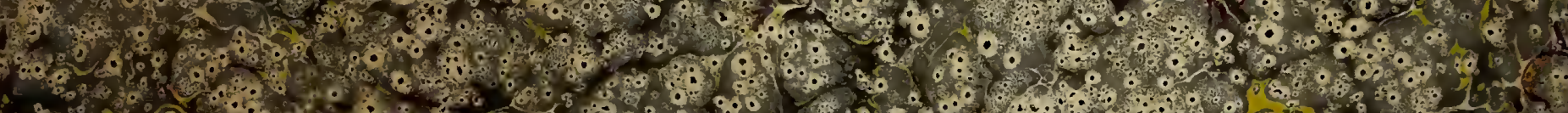

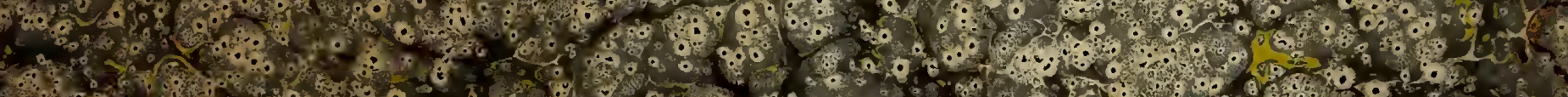

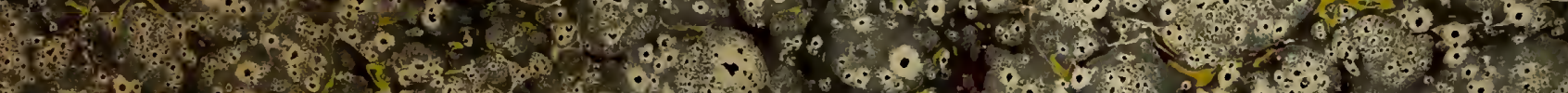

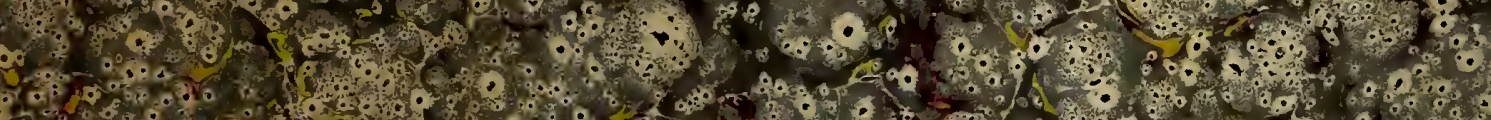

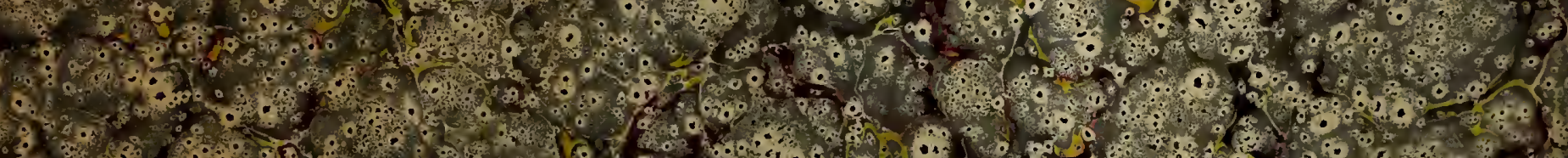

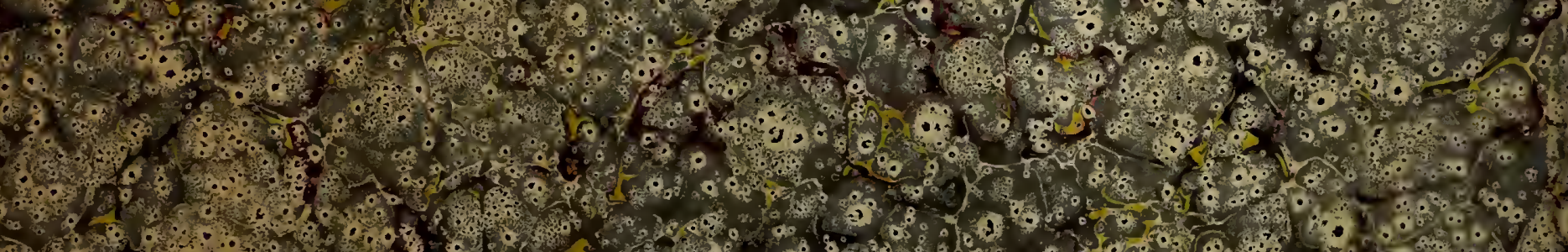

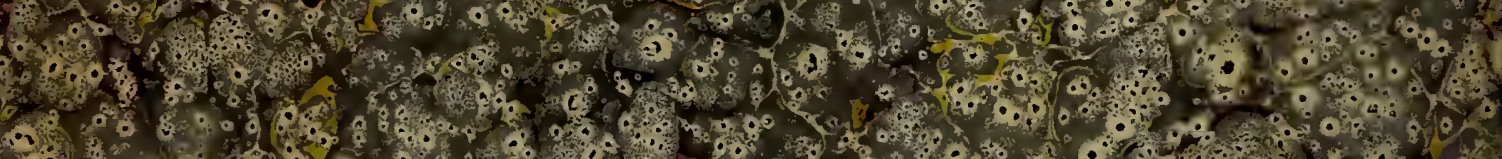

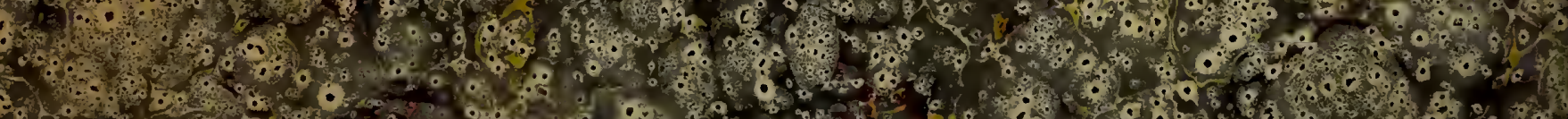

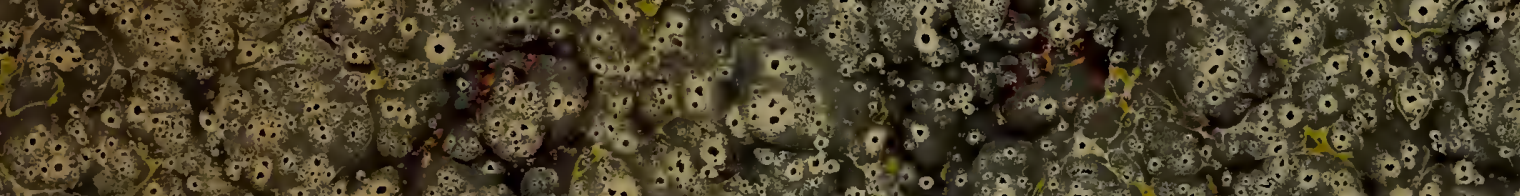

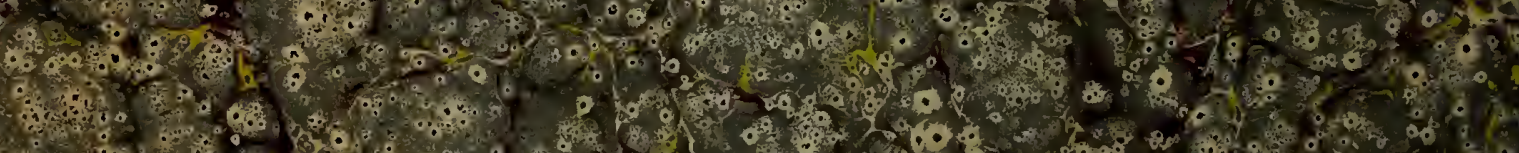

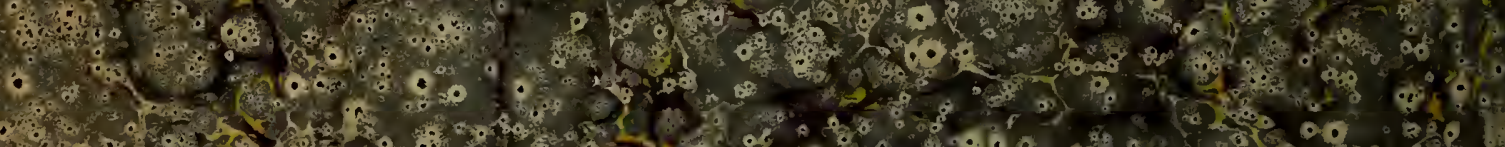

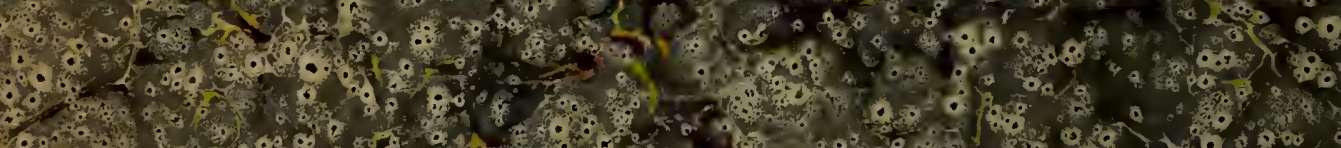

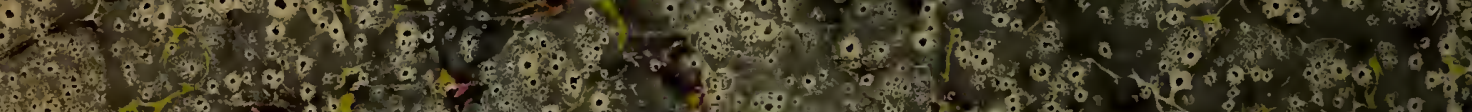

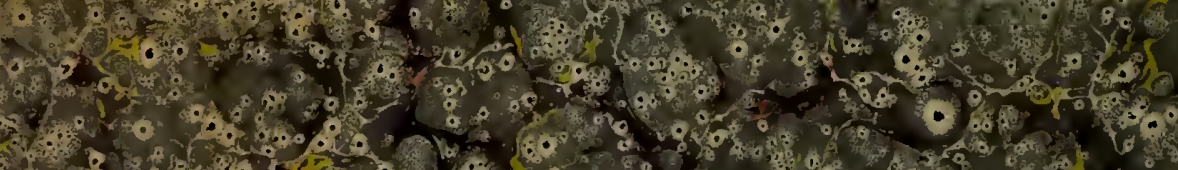
Q $0.0 .0 \%, 8$

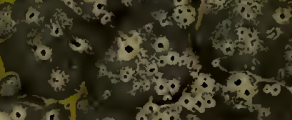
s

- 2 4 a

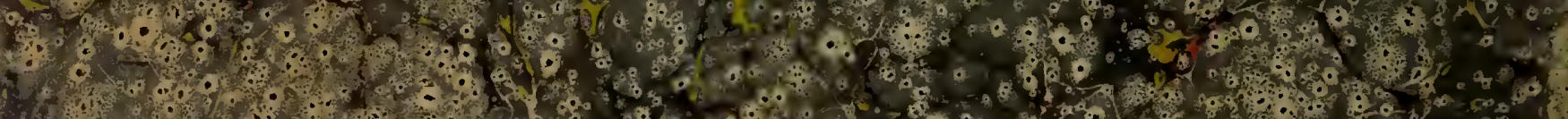
19. $\%(\%, 3)$

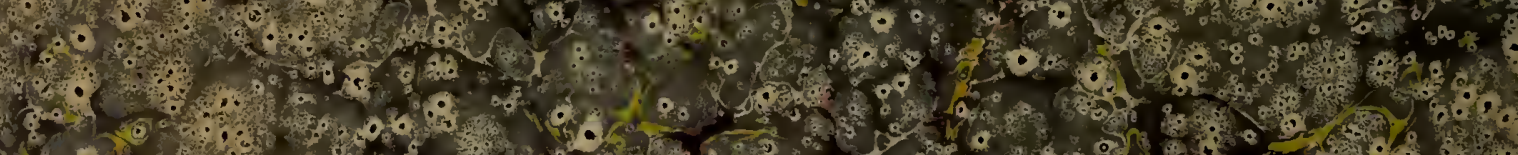

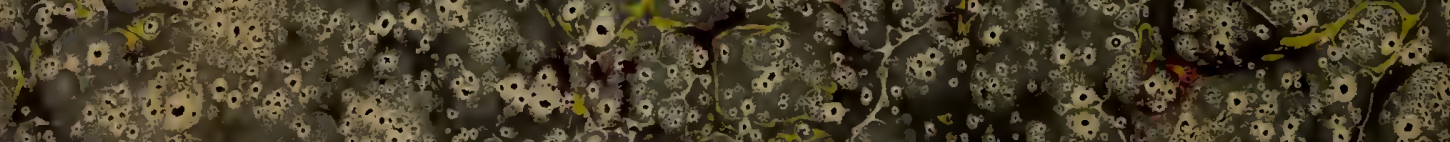

